
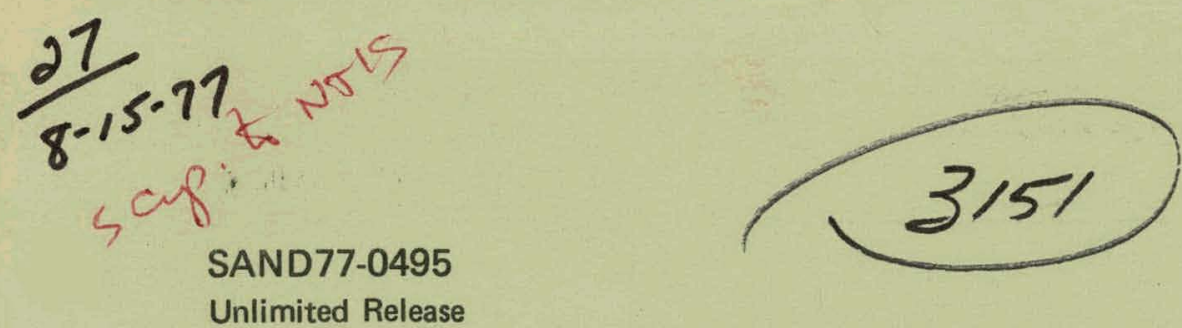

\title{
Location Errors in Time of Arrival (TOA) and Time Difference of Arrival (TDOA) Systems
}

Eugene A. Aronson

Prepared by Sandia Laboratories, Albuquerque New Mexico 87115

and Livermore, Califormia 94550 for the United States Energy Research

and Development Administration under Contract AT (29-1)-789

Printed July 1977

\section{Sandia Laboratories}




\section{DISCLAIMER}

This report was prepared as an account of work sponsored by an agency of the United States Government. Neither the United States Government nor any agency Thereof, nor any of their employees, makes any warranty, express or implied, or assumes any legal liability or responsibility for the accuracy, completeness, or usefulness of any information, apparatus, product, or process disclosed, or represents that its use would not infringe privately owned rights. Reference herein to any specific commercial product, process, or service by trade name, trademark, manufacturer, or otherwise does not necessarily constitute or imply its endorsement, recommendation, or favoring by the United States Government or any agency thereof. The views and opinions of authors expressed herein do not necessarily state or reflect those of the United States Government or any agency thereof. 


\section{DISCLAIMER}

Portions of this document may be illegible in electronic image products. Images are produced from the best available original document. 
Issued by Sandia Laboratories, operated for the United States Energy Research \& Development Administration by Sandia Corporation.

\section{NOTICE}

This report was prepared as an account of work sponsored by the United States Government. Neither the United States nor the United States Energy Research \& Development Administration, nor any of their employees, nor any of their contractors, subcontractors, or their employees, makes any warranty, express or implied, or assumes any legal liability or responsibility for the accuracy, completeness or usefulness of any information, apparatus, product or process disclosed, or represents that its use would not infringe privately owned rights.

Printed in the United States of America

Available from

National Technical Information Service

U. S. Department of Commerce

5285 Port Royal Road

Springfield, VA 22161

Price: Printed Copy $\$ 4.00$; Microfiche $\$ 3.00$ 
SAND 77-0495

Unlimited Release

Printed July 1977

\begin{abstract}
LOCATION ERRORS IN TIME OF ARRIVAL (TOA), AND
TIME DIFFERENCE OF ARRIVAL (TDOA) SYSTEMS
\end{abstract}

Eugene A. Aronson

Applied Mathematics Division 2613

Sandia Laboratories, Albuquerque, NM 87115

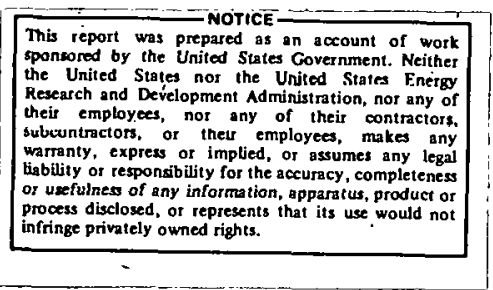

\begin{abstract}
In this report it is shown that the covariance matrix of object location errors is identical for time of arrival (TOA) and time difference of arrival (TDOA) systems if the inverse of the covariance matrix of TOA (TDOA) errors is used as a weighting matrix. Also, with this weighting the location errors statistics do not depend on the particular difference pairs in the TDOA scheme, provided that a complete and nonredundant set is used. If the TOA or TDOA errors are samples of jointly gaussian random variables, this weighting is optimal in the sense of maximum likelihood and minimum varianee. Only rolativo valuod of the woighting noed be lenown for optimality.
\end{abstract}




\section{ACKNOW LEDGMENT}

The author thanks B. M. Marder and H. A. Watts, both of Division 2613, for their assistance in this work. 
CONTENTS

$\underline{\text { Page }}$

Introduction

6

Object Location by TDOA

8

Location Error Statistics

Choice of Data Differences

Proof of the Lemma

13

Proof of Proposition 1

17

Object Location by TOA

Comparison of TOA Errors and TDOA Errors

Conclusions

References 
Introduction

An object at an unknown location generates a pulse of energy at an unknown initial time. This pulse is radiated and detected by a set of sensors. Each sensor estimates the time of arrival (TOA) of the pulse at the sensor. If the velocity of the pulse from object to sensor is known and constant, the TOA minus the initial pulse time is proportional to the distance from object to sensor. If there are more sensors than unknown coordinates of the object, it is possible to estimate the unknown position and the initial time by least-squares techniques.

Since the initial time itself is not generally of interest, it is common to remove it by taking time differences of arrival (TDOA) and solving a system of equations of order one less than with TOA in the least-squares sense.

In general, the TOA data contain errors; and hence any data reduction scheme produces estimates of the object location that also contain errors. Since the errors in the TOA estimates are not generally the same statistically, it is usually advantageous to use a weighting scheme to reduce object location errors.

In particular, if the data errors are jointly gaussian random variables with zero mean, which is not a generally restrictive assumption, the maximum-likelihood, minimum-variance estimate of the location errors is obtained if the weighting matrix is the inverse of the covariance matrix of the TOA errors or of the TDOA errors, if such a scheme is used.

In this report we prove that if the aforementioned weighting matrix is used, the object location error statistics are identical for both TOA and TDOA schemes. Also, these statistics are the same no matter what data difference pairs are chosen for the TDOA method, provided that an independent, nonredundant set of differences is used.

The statement above is actually proved only for the very typical case when the TOA errors are independent random variables, but we feel it is valid for correlated TOA errors.

While our interest here is in locating an object by TOA or TDOA, the statement is just as valid for estimating any unknowns from data when a data model function--in our case, distance-is compared to data in the least-squares sense. All that is required is that the model function be continuous and differentiable in the unknowns. 
In the following material we use capital letters to denote matrices or vectors and lower case letters for scalars. All vectors are column vectors unless expressed as a transpose. The superscript $\mathrm{T}$ means "transpose."

Object Location by TDOA

It is desired to estimate the location of an object. At some unknown time a pulse of energy is generated at the object. This pulse radiates and is detected by a set of sensors at fixed, known locations. We estimate a TOA of the pulse at each sensor. By operating on the TDOA between pairs of sensors, we estimate the object position.

Let the (unknown) object position be

$$
P=\{x, y, z\}
$$

Let there be $\mathrm{N}+1$ sensors, and let the known position of the $\underline{n}$ th sensor be

$$
P_{n}=\left\{x_{n}, y_{n}, z_{n}\right\} ; n=1,2, \ldots, N+1
$$

The distance from object to the nth sensor is

$$
r_{n}=\sqrt{\left(x-x_{n}\right)^{2}+\left(y-y_{n}\right)^{2}+\left(z-z_{n}\right)^{2}} .
$$

If $t_{n}$ is the TOA at the nth sensor and $v_{p}$ is the (constant) veloclty of the pulse, we must find $P$ Go ao tó sutiafy the PJ equalluns

$$
\begin{aligned}
& r_{m_{1}}-r_{m_{2}}=v_{p}\left(t_{m_{1}}-t_{m_{2}}\right) . \\
& m=1,2, \ldots, N+1,1 \leq m_{1}, m_{2} \leq N .
\end{aligned}
$$

If all three coordinates of $\mathrm{P}$ are unknown, at least four sensors are required. If any two conrdinälés dr't unknown, at least three sensors are needed, etc.

We assume that whatever pairings of data are used in Eq. (1), exactly $N$ nonredundant, independent time differences are employed. These conditions are determined hy examining an $\mathrm{N}$ by $\mathrm{N}+1$ test matrix. Let all elements of the test matrix be zero except for +1 in the $\underline{m}^{\text {th }}$ row and $m_{1}$ column and -1 in the $\underline{m}$ th row and $m_{2}$ column. If the test matrix has rank $N$, the differences are independent and nonredundant. 
In general there are more data differences than unknowns, and Eqs. (1) are solved in the least-squares sense. It is convenient to use matrix notation. Let $R$ and $D$ be $N$-dimensional . column vectors

$$
\begin{aligned}
& R=\left\{r_{1}-r_{1}, \ldots, r_{m_{1}}-r_{m_{2}}, \ldots, r_{N_{1}}-r_{N_{2}}\right\} ; \\
& D=\left\{v_{p}\left(t_{1}-t_{1}\right), \ldots, v_{p}\left(t_{N_{1}}-t_{N_{2}}\right)\right\} .
\end{aligned}
$$

If $\mathrm{W}$ is an $\mathrm{N}-\mathrm{b} y-\mathrm{N}$ symmetric weighting matrix, we wish to find $\mathrm{P}$ so as to minimize the scalar

$$
v=(R-D)^{T} W(R-D)
$$

Minimization of $\mathrm{v}$ is usually done by Newton iteration. Let $\mathrm{A}$ be the $\mathrm{N}-\mathrm{by}-3 \mathrm{matrix}$

$$
A=\left[\begin{array}{ccc}
a_{1}-a_{1} & b_{1}-b_{1} & c_{1}-c_{12} \\
\vdots & \vdots & \vdots \\
a_{m_{1}}-a_{m_{2}} & b_{m_{1}}-b_{m_{2}} & c_{m_{1}}-c_{m_{2}} \\
\vdots & \vdots & \vdots \\
a_{N_{1}}-a_{N_{2}} & b_{N_{1}}-b_{N_{2}} & c_{N_{1}}-c_{N_{2}}
\end{array}\right]=\frac{d R}{d P}
$$

where

$$
\begin{aligned}
& a_{n}=\left(x-x_{n}\right) / r_{n}, \\
& b_{n}=\left(y-y_{n}\right) / r_{i 1}, \\
& c_{n}=\left(z-z_{n}\right) / r_{n} .
\end{aligned}
$$

Of course, $A$ is $\mathrm{N}$ by 2 if there are only two unknowns, etc. If $\mathrm{P}^{\mathrm{P}}$ is the pth estimate of $P$, and $A^{\mathrm{p}}$ and $R^{p}$ are $A$ and $R$, respectively, evaluated at $P=P^{p}$, the iteration is

$$
P^{p+1}=P^{p}+\left(A^{p T}{ }^{p A}\right)^{-1}{ }^{p T} W\left(R^{p}-n\right)
$$

If the data errors are "small" and the system is "well conditioned," convergence is rapid and the location errors are small. The condition of the system is determined by the condition of the matrix ${ }^{p T}{ }^{p A}{ }^{p}$. Note that this matrix depends only on the system geometry and the choice 
of $\mathrm{W}$, not on the data or their errors. The iteration is usually terminated after a fixed number of iterates or when the change in location estimates becomes less than some preassigned value. Some discussion of location estimate errors is in Ref. 1.

\section{Location Error Statistics}

It is of interest to estimate the errors in the object location for any set of object and sensor geometry. If the data errors; i.e., the TOA errors, are "small" and the system is "well conditioned," then it can be shown that the object location errors depend linearly on the data errors. ${ }^{1}$ Let $P_{e}$ be the errors in the estimates of the object location, and let $D_{e}$ be the data errors. For TDOA, $\mathrm{D}_{e}$ is the vector of data error differences; fur TOA it is the vector of the data errors themselves. We get

$$
P_{e}=B^{-1} A^{T} W D_{e}
$$

where

$$
B=A^{T} W A
$$

Let $E[$.$] denote expected value. In general we may assume that E\left[D_{e}\right]=0$ and hence

$$
E\left[P_{e}\right]=0
$$

If $\mathrm{C}$ is the covariance matrix of the dal'd errors,

$$
C=E\left[D_{e^{D}} e^{T}\right]
$$

then the covariance matrix of the position errors is

$$
\Gamma_{c}-E\left[P_{e} P_{e}^{T}\right]=R^{-1} A^{T} W_{C W A B}{ }^{-1}
$$

If the data errors are jointly gaussian randun valiables, thon an optimum rhnice of $W$ is

$$
\mathrm{W}=\mathrm{C}^{-1}
$$

This choice yields the maximum-likelihood, minimum-variance estimate of the object location. ${ }^{2}$ In this case Eq. (7) simplifies to

$$
P_{c}=B^{-1}
$$


The gaussian assumption is reasonable for most systems. If the data errors are jointly gaussian, then, from Eq. (4) the location errors are also jointly gaussian and all their statistics are known from their mean and covariances. The results presented thus far, and indeed all results in this paper, are valid whether or not the data errors are gaussian. The gaussian property assures only that the choice $\mathrm{W}=\mathrm{C}^{-1}$ is optimal in the maximum-likelihood, minimum-variance sense.

\section{Choice of Data Differences}

We now arrive at the first of the two salient points of this report. We prove the following:

\section{Proposition 1}

If the TOA data errors are independent random variables with zero means and the weighting matrix is chosen as the inverse of the covariance of the data difference errors, then the covariance matrix of the position errors is independent of the particular choice of difference pairs--provided, of course, that a set of independent, nonredundant differences is used.

It is likely that the proposition is also true if the TOA errors are correlated, but the proof of this statement is too tedious to pursue here. The independence of TOA errors is usually valid since the sensors are typically separated physically and subject to independent noise processes.

The proof is quite lengthy and is broken into two sections. First, an important lemma is proved that states that Proposition 1 is valid if any one TOA appears in all differences; that is, the value of $P_{c}$ does not depend on which TOA is common to all differences. 'l'he main proof then follows by induction. We show that the proposition is true for threc sensors; we then assume it true for $\mathrm{N}$ sensors. Now, if an additional sensor is introduced, we show that $\mathrm{P}_{\mathrm{c}}$ does not depend on with which of the original $\mathrm{N}$ sensors this new TOA data is paired. Hence, the proposition is true for any number of sensors and any set of pairings.

Before proceeding, we introduce some notation and present two important theorems.

Let the TOA error from the nth sensor be $t_{e n}$. Then

$$
\mathrm{E}\left[\tilde{t}_{\text {en }}\right]=\mathrm{E}\left[\mathrm{t}_{\text {en }} \mathrm{t}_{\mathrm{em}}\right]=0, \mathrm{~m} \neq \mathrm{n}
$$

The TOA error variance is

$$
v_{n}=v_{p}^{2} E\left[t_{e n}^{2}\right]
$$


We assume that $\nu_{\mathrm{n}}>0$ for all $\mathrm{n}$ and define

$$
\mathrm{g}_{\mathrm{n}}=1 / \nu_{\mathrm{n}}
$$

Theorem 1: (Ref. 3) -- If $\mathrm{G}^{-1}$ exists and

$$
C=G+U V^{T} \text {. }
$$

where $U$ and $V$ are column vectors, then

$$
\begin{aligned}
\mathrm{C}^{-1} & =\mathrm{G}^{-1}-\lambda \mathrm{G}^{-1} \mathrm{UV}^{\mathrm{T}} \mathrm{G}^{-1}, \\
\lambda & =1 /\left(1+\mathrm{VG}^{-1} \mathrm{U}\right) .
\end{aligned}
$$

Theorem 2: (Ref. 4) -- Suppose the matrix $\hat{\mathrm{C}}$ is composed of submatrices of the indicated orders

$$
\hat{\mathrm{C}}=\left[\begin{array}{ccc}
\mathrm{C} & 1 & \mathrm{C}_{12} \\
(\mathrm{~N} \times \mathrm{N}) & 1 & (\mathrm{~N} \times \mathrm{M}) \\
\hdashline--- & - & --- \\
\mathrm{C}_{21} & : & \mathrm{C}_{22} \\
(\mathrm{M} \times \mathrm{N}) & 1 & (\mathrm{M} \times \mathrm{M})
\end{array}\right],
$$

and $\mathrm{C}$ and $\mathrm{Q}=\mathrm{C}_{22}-\mathrm{C}_{21}\left(\mathrm{WC}_{12}\right)$ are nonsingular, where $\mathrm{C}^{-1}=\mathrm{W}$. Then

$$
\hat{\mathrm{C}}^{-1}=\left[\begin{array}{lll}
\mathrm{W}_{11} & : & \mathrm{W}_{12} \\
(\mathrm{~N} \times \mathrm{N}) & : & (\mathrm{N} \times \mathrm{M}) \\
\hdashline \mathrm{W}_{21} & : & \mathrm{W}_{22} \\
(\mathrm{M} \times \mathrm{N}) & : & (\mathrm{M} \times \mathrm{M})
\end{array}\right]
$$

dind

$$
\begin{aligned}
& W_{11}=W+\left(W_{13}\right) Q^{-1}\left(r_{21} W\right) \\
& W_{12}=-\left(W_{12}\right) Q^{-1}, \\
& W_{21}=-Q^{-1}\left(C_{21} W\right) \\
& W_{22}=Q^{-1} .
\end{aligned}
$$


We shall use Theorem 2 only with $M=1$ and $\hat{C}$ symmetric. In our case $Q$ is a scalar, $\mathrm{C}_{21}=\mathrm{C}_{21}^{\mathrm{T}}$, and $\mathrm{C}$ and $\mathrm{W}$ are symmetric. We get

$$
\begin{aligned}
\mathrm{Q} & =\mathrm{q}=\mathrm{c}_{22}-\mathrm{C}_{12}^{\mathrm{T}} \mathrm{WC}_{12}, \\
\mathrm{w}_{11} & =\mathrm{w}+\left(\mathrm{WC}_{12}\right)\left(\mathrm{WC}_{12}\right)^{\mathrm{T}} / \mathrm{q}, \\
\mathrm{w}_{12} & =\mathrm{w}_{21}^{\mathrm{T}}=-\left(\mathrm{WC}_{12} / \mathrm{q},\right. \\
\mathrm{w}_{22} & =1 / \mathrm{q} .
\end{aligned}
$$

Proof of the Lemma

Lemma: If one sensor appears in all differences, then the values of $B$ and hence of its inverse $P_{c}$ does not depend on which sensor is the common one--provided the TOA errors are independent random variables and $\mathrm{W}=\mathrm{C}^{-1}$.

Suppose there are $\mathrm{N}+1$ sensors. Choose the $(\mathrm{N}+1)$ - st sensor as the common one, The $\mathrm{N}$ by $\mathrm{N}$ covariance matrix of the data difference errors is

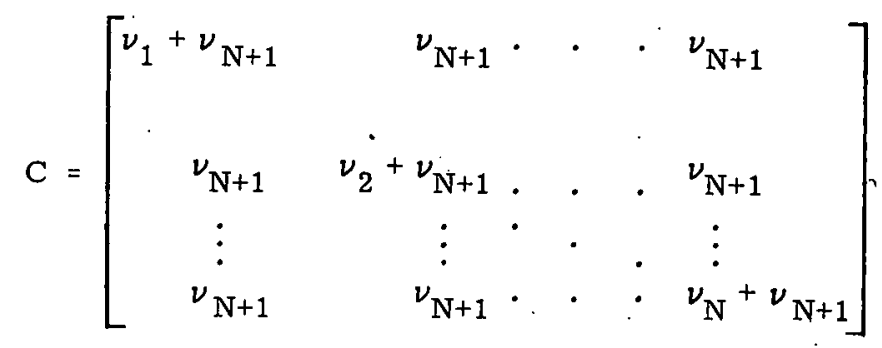

This matrix is clearly of the form of Theorem 1 , with

$$
\mathrm{G}=\left[\begin{array}{llll}
\nu_{1} & & & \\
& & & \\
& \nu_{2} & \\
& & \ddots & \\
& 0 & & \nu_{N}^{\prime}
\end{array}\right]
$$

and

$$
\begin{aligned}
& \mathrm{U}=v_{\mathrm{N}+1}\{1,1, \ldots, 1\}, \\
& \mathrm{V}=\{1,1, \ldots, 1\},
\end{aligned}
$$


Thus,

$$
\mathrm{G}^{-1}=\left[\begin{array}{ccc}
\mathrm{g}_{1} & & \\
& & \\
& \mathrm{~g}_{2} & \\
& & \ddots \\
\mathrm{g}_{\mathrm{N}}
\end{array}\right]
$$

and

$$
\lambda=1 /\left(1+u_{N+1} \sum_{n=1}^{N} g_{n 1}\right) .
$$

If we defino

$$
s=\sum_{n=1}^{N+1} g_{n} \text {. }
$$

then $\lambda=1 / s \nu_{N+1}$. Note that the value of $s$ does not depend on which TOA was chosen as the common one. Now, from Theorem 1, the optimum weighting matrix is

$$
\mathrm{W}=\mathrm{c}^{-1}=\left[\begin{array}{cccc}
\mathrm{g}_{1}-\mathrm{g}_{1}^{2} / \mathrm{s} & -\mathrm{g}_{1} \mathrm{~g}_{2} / \mathrm{s} & \ldots & -\mathrm{g}_{1} \mathrm{~g}_{\mathrm{N}} / \mathrm{s} \\
-\mathrm{E}_{1} \mathrm{~g}_{2} / \mathrm{s} & \mathrm{g}_{2}-\mathrm{g}_{2}^{2} / \mathrm{s} & \ldots & -\mathrm{g}_{2} \mathrm{~g}_{\mathrm{N}} / \mathrm{s} \\
\vdots & \vdots & \ddots & \vdots \\
-\mathrm{g}_{1} \mathrm{~g}_{\mathrm{N}} / \mathrm{s} & -\mathrm{g}_{2} \mathrm{~g}_{N} / \mathrm{s} & \cdots & \mathrm{g}_{\mathrm{N}}-\mathrm{g}_{\mathrm{N}}^{2} / \mathrm{s}
\end{array}\right] \text {. }
$$

For simplicity in the exposition we will let the $\mathrm{z}$ coordinate of the object be known. It will be shown that this is not a restrictive assumption. For this situation with two unknowns the $A$ matrix is $\mathrm{N}$ by 2 . Its form for the lemma is

$$
A=\left[\begin{array}{cc}
a_{N+1}-a_{1} & b_{N+1}-b_{1} \\
a_{N+1}-a_{2} & b_{N+1}-b_{2} \\
\vdots & \vdots \\
a_{N+1}-a_{N} & b_{N+1}-b_{N}
\end{array}\right] .
$$


We now compute $B=A^{T}$ W. First,

$$
W A=\frac{1}{s}\left[\begin{array}{cc}
g_{1}\left[\left(a_{N+1}-a_{1}\right) s-\sum_{n} g_{n}\left(a_{N+1}-a_{n}\right)\right] & g_{1}\left[\left(b_{N+1}-b_{1}\right) s-\sum_{n} g_{n}\left(b_{N+1}-b_{n}\right)\right] \\
\vdots & \vdots \\
g_{N}\left[\left(a_{N+1}-a_{N}\right) s-\sum_{n} g_{n}\left(a_{N+1}-a_{n}\right)\right] & g_{N}\left[\left(b_{N+1}-b_{N}\right) s-\sum_{n} g_{n}\left(b_{N+1}-b_{n}\right)\right]
\end{array}\right] .
$$

All the sums in WA are from 1 to $N$; but, since $a_{N+1}-a_{N+1}=0$ and $b_{N+1}-b_{N+1}=0$, we can take all sums from 1 to $\mathrm{N}+1$ without affecting the result. Consider the element in the $\underline{m}$ th row and first column of WA,

$$
\begin{aligned}
g_{m}\left[\left(a_{N+1}-a_{m}\right) s-\sum_{n=1}^{N} g_{n}\left(a_{N+1}-a_{n}\right)\right] & =g_{m}\left(a_{N+1} s-a_{m} s-a_{N+1} s+\sum_{n=1}^{N+1} g_{n} a_{n}\right) \\
& =g_{m}\left(\sum_{n=1}^{N+1} g_{n} a_{n}-a_{m} s\right) .
\end{aligned}
$$

Now, define

$$
h_{a}=\sum_{n=1}^{N+1} g_{n} a_{n} \text { and } \dot{h}_{b}=\sum_{n=1}^{N+1} g_{n} b_{n} \text {. }
$$

We note that $h_{a}$ and $h_{b}$, like $s$, are independent of the common index. We now get

$$
W A=\frac{1}{s}\left[\begin{array}{cc}
g_{1}\left(h_{a}-a_{1} s\right) & g_{1}\left(h_{b}-b_{1} s\right) \\
\vdots & \vdots \\
g_{N}\left(h_{a}-a_{N} s\right) & g_{N}\left(h_{b}-b_{N} s\right)
\end{array}\right]
$$

The matrix $B$ is seen to be

$$
B=\frac{1}{s}\left[\begin{array}{ll}
\sum_{n}\left(a_{N+1}-a_{n}\right) g_{n}\left(h_{a}-a_{n} s\right) & \sum_{n}\left(a_{N+1}-a_{n}\right) g_{n}\left(h_{b}-b_{n} s\right) \\
\sum_{n}\left(b_{N+1}-b_{n}\right) g_{n}\left(h_{a}-a_{n} s\right) & \sum_{n}\left(b_{N+1}-b_{n}\right) g_{n}\left(h_{b}-b_{n} s\right)
\end{array}\right]
$$

These sums can be taken from 1 to $N+1$. 
We denote the elements of $B$ as

$$
\mathrm{B}=\left[\begin{array}{ll}
\beta_{11} & \beta_{12} \\
\beta_{21} & \beta_{22}
\end{array}\right] \text {. }
$$

Then,

$$
\begin{aligned}
\beta_{11} & =\left[a_{N+1}\left(h_{a} \sum g_{n}-s \sum g_{n} a_{n}\right)-h_{a} \sum g_{n} a_{n}+s \sum g_{n} a_{n}^{2}\right] / s \\
& =\left[a_{N+1}\left(h_{a} s-s h_{a}\right)-h_{a}^{2}+s \sum g_{n} a_{n}^{2}\right] / s .
\end{aligned}
$$

By similar operations on the elements of $\mathrm{B}$ we conclude that

$$
\begin{aligned}
& \beta_{11}=\sum_{n=1}^{N \cdot 1+1} y_{n} a_{n}^{2}-1_{a}^{2} / s, \\
& \beta_{12}=\beta_{21}=\sum_{n=1}^{N+1} g_{n} a_{n} b_{n}-h_{a} h_{b} / s, \\
& \beta_{22}=\sum_{n=1}^{N+1} g_{n} b_{n}^{2}-h_{b}^{2} / s .
\end{aligned}
$$

Thus the elements of $B$ do not depend on the choice of common TOA, and the lemma is proved.

If A were an N-by-3 matrix; 1.e., If three coordinates of the object were unknown, then the third column would contain terms of the form $c_{N+1}-c_{n}, n=1,2, \ldots, N$, and B would be 3 by 3 . However, the new elements of $\mathrm{B}--\beta_{13}=\beta_{31}, \beta_{33}=\beta_{33}$, and $\beta_{33}--$ can be evaluated merely by replacing $a$ or b by $c$ in Eqs. (15). In general, therefore, the lemma is true and Eqs. (15) are valid for any number of unknowns.

Also, proof of the lemma did not use the particular forms of $a_{n}$ and $b_{n}$ specified in the TDOA method. Thus the lemma is true for any least-squares scheme that minimizes

$$
v=(R-D)^{T} W(R-D)
$$

where $R$ is the vector of pairs of function differences and $D$ is the vector of independent data differences. All that is required is that $R$ satisfy the usual continuity and differentiability 
criteria. Of course, we must have $\mathrm{W}=\mathrm{C}^{-1}$, and the lemma has been proved true only if the individual data errors are mutually independent.

\section{Proof of Proposition 1}

We are now ready to prove Proposition 1. As before we will use only two unknowns, say $x$ and $y$, and then show that Proposition 1 is valid for any number of unknowns.

Suppose there are data from three sensors. The proposition is true from the lemma since two independent differences using three data values must have one data value in common. Now, assume the proposition is true for $N>3$. We obtain data from an $N+1$ sensor and introduce the additional dat.a difference $v_{p}\left(t_{N+1}-t_{k}\right), k=1,2, \ldots . N$ into the computation of $B$. Clearly the new set of $\mathrm{N}$ differences is independent and nonredundant. If the value of $B$ is now independent of the choice of $k$, then the proposition is true for all $\mathrm{N}$ by induction.

Since the proposition is assumed true for $\mathrm{N}$ sensors, we may without loss of generality take the original $\mathrm{N}+1$ differences with the data from one sensor as a common element. Let the common data element be the Nth one. If $k=N$, the proposition is true from the lemma. Thus we restrict $\mathrm{k}=1,2, \ldots, \mathrm{N}-1$.

Thè matrices $\mathrm{C}$ and $\mathrm{W}$ with $\mathrm{N}$ data values are given by. Eqs. (11) and (13), respectively, with $\mathrm{N}$ replaced by $\mathrm{N}-1$. With the additional data we must evaluate a new $\mathrm{B}$ matrix, viz,

$$
B=\hat{A}^{T} \hat{W} \hat{A}
$$

where

$$
\begin{gathered}
\hat{A}=\left[\begin{array}{c}
A \\
-\hat{A}_{k}
\end{array}\right]=\left[\begin{array}{cc}
a_{N}-a_{1} & b_{N}-b_{1} \\
\vdots & \vdots \\
a_{N}-a_{N-1} & b_{N}-b_{N-1} \\
-------- & -b_{N+1}-a_{k} \\
a_{N+1}-b_{k}
\end{array}\right], \\
\hat{W}=\hat{C}^{-1},
\end{gathered}
$$

and

$$
\hat{\mathrm{C}}\left[\begin{array}{lll}
\mathrm{C} & \mathrm{C}_{12} \\
- & - & - \\
\mathrm{C}_{12}^{\mathrm{T}} & \mathrm{C}_{2.2}
\end{array}\right] \text {, }
$$

ख. 
with

$$
\begin{aligned}
& c_{12}=\left\{0,0, \ldots . \nu_{k}, 0, \ldots .0\right\} \\
& c_{22}=\nu_{N+1}+\nu_{k} .
\end{aligned}
$$

The only nonzero entry in $\mathrm{C}_{12}$ is in the kth column. The matrices $\hat{\mathrm{C}}$ and $\hat{\mathrm{W}}$ are $\mathrm{N}$ by $\mathrm{N}$, $\mathrm{C}_{12}$ is $\mathrm{N}-1$ by 1 , and $\hat{\mathrm{A}}$ is $\mathrm{N}$ by 2 .

Since $\hat{C}$ is symmetric, $\hat{W}$ is symmetric. We have

$$
\hat{\mathrm{w}}=\left[\begin{array}{c:c}
\mathrm{w}_{11} & \mathrm{w}_{12} \\
\hdashline \mathrm{w}_{12}^{\mathrm{T}} & \hat{\mathrm{w}}_{22}
\end{array}\right] \text {, }
$$

and by standard matrix operations,

$$
\begin{aligned}
B & =A^{T} W_{11} A+A^{T} W_{12} A_{k}+A_{k}^{T} W_{12}^{T} A+A_{k}^{T} A_{k} \hat{w}_{22} \\
& =Q_{1}+Q_{2}+Q_{2}^{T}+Q_{3},
\end{aligned}
$$

where Eq. (16) defines the $Q_{i}$. Note that $Q_{1} \cdot Q_{\dot{2}}+Q_{2}^{T}$, and $Q_{3}$ 2ro oymmctric.

Denote by the superscript $k$ that part of a matrix that depends on $k$. For example, we can write $R=B^{\prime}+B^{k}$. Tho $B^{k}$ dcpends in $k$ and the $F^{\prime}$, does not. We wish to prove that $B^{k}=0$.

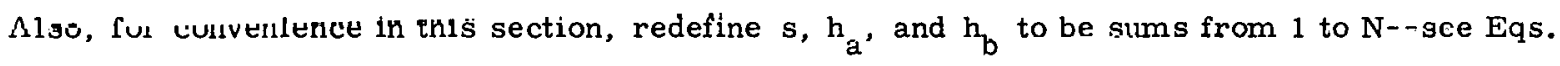
(12) and (14).

We now apply Theorem 2. The scalar $q$ is

$$
q=1 / \hat{w}_{22}=\nu_{N+1}+\nu_{k}-\nu_{k}^{2} w_{k k},
$$

where $\mathrm{w}_{\mathrm{kk}}$ is the $\underline{\mathrm{k}}$ th diagonal element of $\mathrm{W}$. Hence,

$$
\begin{aligned}
q & =v_{\mathrm{N}+1}+\nu_{\mathrm{k}}-\nu_{\mathrm{k}}^{2}\left(\mathrm{~g}_{\mathrm{k}}-\mathrm{g}_{\mathrm{k}}^{2} / \mathrm{s}\right) \\
& =\nu_{\mathrm{N}+1}+\nu_{\mathrm{l}}-\nu_{\mathrm{k}}+1 / \mathrm{s}=\nu_{\mathrm{N}+1}+1 / \mathrm{s} .
\end{aligned}
$$

and $\mathrm{q}$ is independent of $\mathrm{k}$. The vector $\mathrm{WC}_{12}$ is the kth column of $\mathrm{W}$ multiplied by $\nu_{\mathrm{k}}$. We have 


$$
\begin{aligned}
\mathrm{WC}_{12} & =v_{\mathrm{k}}\left\{-\mathrm{g}_{1} \mathrm{~g}_{\mathrm{k}} \ldots \ldots-\mathrm{g}_{\mathrm{k}}^{2}+\mathrm{sg}_{\mathrm{k}} \ldots \ldots-\mathrm{g}_{\mathrm{N}-1} \mathrm{~g}_{\mathrm{k}}\right\} / \mathrm{s} \\
& =\left\{-\mathrm{g}_{1},-\mathrm{g}_{2}, \ldots,-\mathrm{g}_{\mathrm{k}}+\mathrm{s}, \ldots,-\mathrm{g}_{\mathrm{N}-1}\right\} / \mathrm{s}
\end{aligned}
$$

The first term of Eq. (16) is

$$
Q_{1}=A^{T} W A+A^{T}\left(W_{12}\right)\left(W_{12}\right)^{T} A / q
$$

Denoting $\left(\mathrm{WC}_{12}\right)\left(\mathrm{WC}_{12}\right)^{\mathrm{T}} / \mathrm{q}$ as $\mathrm{Q}_{1}^{\prime}+\mathrm{Q}_{1}^{\prime \prime}$ we can write

$$
\begin{aligned}
Q_{1}^{\prime} & {\left[\begin{array}{ccc}
g_{1}^{2} & \cdots & g_{1} g_{N-1} \\
\vdots & & \vdots \\
g_{1} g_{N-1} & \cdots & g_{N-1}^{2}
\end{array}\right] / s^{2} q } \\
Q_{1}^{\prime \prime} & {\left[\begin{array}{cccccc}
0 & 0 & \ldots & -g_{1} & \cdots & 0 \\
0 & 0 & \ldots & -g_{2} & \cdots & 0 \\
\vdots & \vdots & & \vdots & & \vdots \\
-g_{1} & -g_{2} & \ldots & s-2 g_{k} & \cdots & -g_{N-1} \\
\vdots & \vdots & & \vdots & & \vdots \\
0 & 0 & \ldots & -g_{N-1} & \cdots & 0
\end{array}\right] }
\end{aligned}
$$

that is, the only nonzero elements of $Q_{1}^{\prime \prime}$ are in its kth row and kth column. Now,

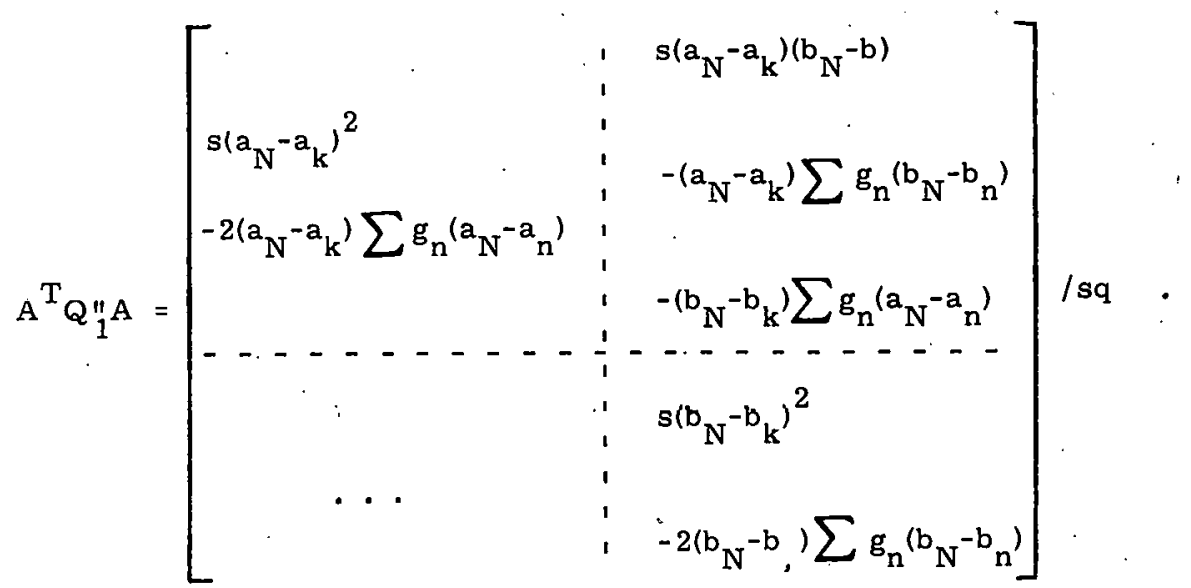


The sums in the expression above are over $\mathrm{n}$ and may be taken from 1 to $N$. Since $A$, W, and $Q_{1}^{\prime}$ do not depend on $k$, as well as certain terms in $A^{T}{ }_{1}^{\prime \prime} A$,

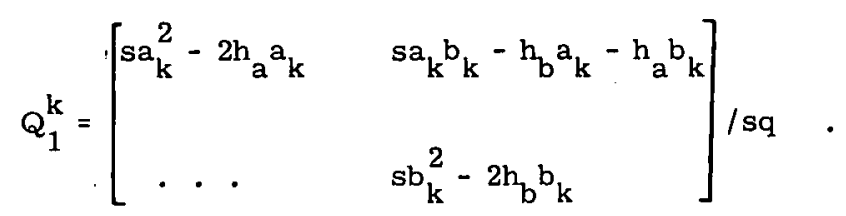

Taking the next term of Eq. (16),

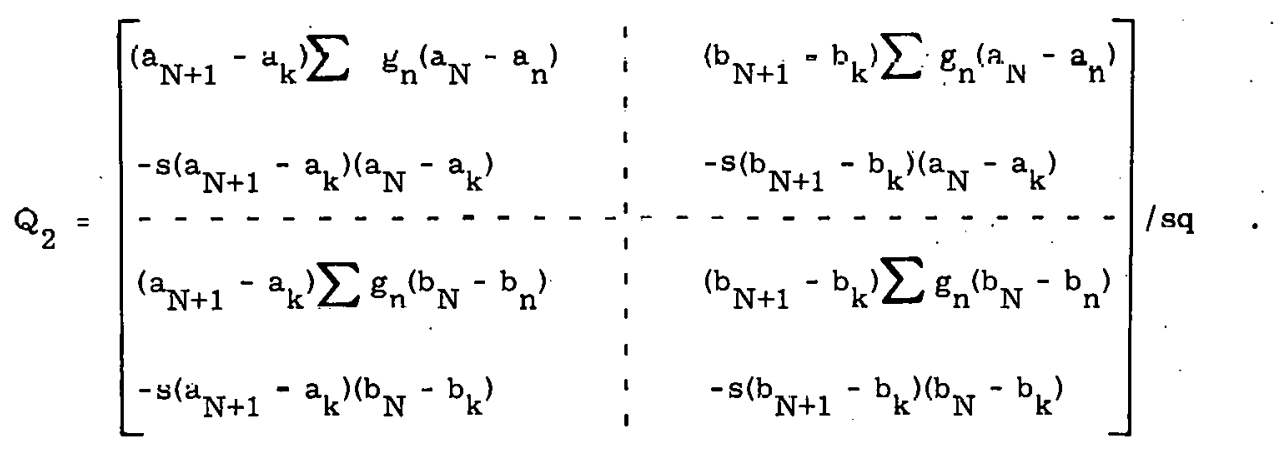

As usual the sums are on $n$ from 1 to $N$. Thus,

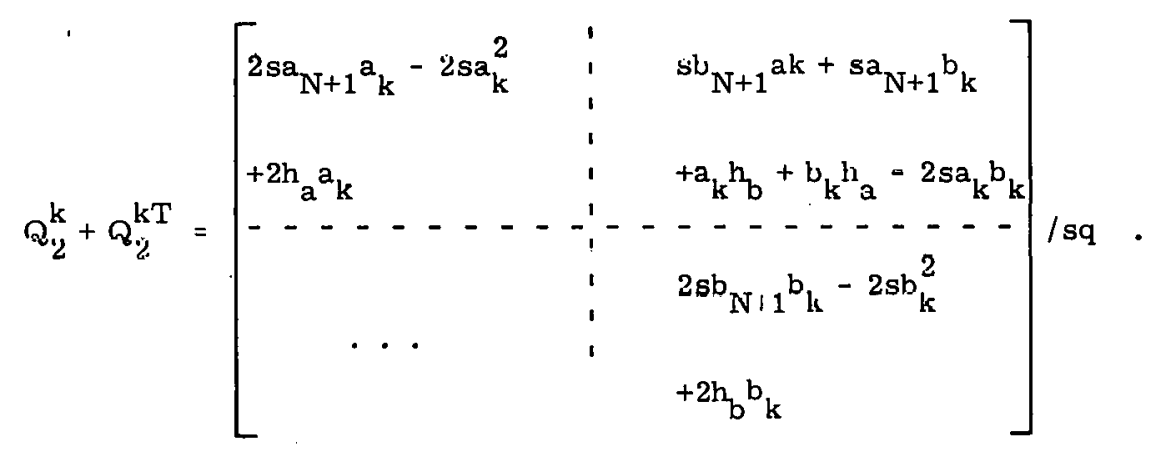

Finally,

$$
\AA_{3}=\left[\begin{array}{cc}
s\left(a_{N+1}-a_{k}\right)^{2} & s\left(a_{N+1}-a_{k}\right)\left(b_{N+1}-b_{k}\right) \\
\cdots & s\left(b_{N+1}-b_{k}\right)^{2}
\end{array}\right] / s q,
$$

and

$$
Q_{3}^{k}=\left[\begin{array}{cc}
s a_{k}^{2}-2 s a_{N+1} a_{k} & s a_{k} b_{k}-s a_{N+1} b_{k}-s b_{N+1} a_{k} \\
\cdots & s b_{k}^{2}-2 s b_{N+1} b_{k}
\end{array}\right] / s q
$$


Combining Eqs. (17), (18), and (19) gives

$$
\mathrm{B}^{\mathrm{k}}=\mathrm{Q}_{1}^{\mathrm{k}}+\mathrm{Q}_{2}^{\mathrm{k}}+\mathrm{Q}_{2}^{\mathrm{kT}}+\mathrm{Q}_{3}^{\mathrm{k}}=0
$$

Thus Proposition 1 is true for two unknowns. However, because $a$ and $b$ appear only in pairs and $B$ is symmetric, the result is valid if either $a$ or $b$ is replaced by $c$, etc. Thus the proposition is true for any number of unknowns.

Again we note that the result does not depend on the particular form of $a$ and $b$ and therefore it is valid for any kind of least-squares data difference scheme, with the usual caveats.

\section{Object Location by TOA}

Instead of locating the object by the TDOA method, we can introduce an additional unknown $u$ and use the TOA data directly, without differences. Suppose the pulse is initiated at the object at the unknown time $t_{0}$. Then $u=v_{p_{0}} t_{0}$ The new vector of unknowns is

$$
P_{u}=\{x, y, z, u\}
$$

Let there be TOA data from $\mathrm{N}$ sensors. The scalar to be minimized is

$$
v_{u}=\left(R_{u}-D_{u}\right)^{T} w_{u}\left(R_{u}^{\prime}-D_{u}\right),
$$

where $R_{u}$ and $D_{u}$ are the $N$-dimensional vectors

$$
\begin{aligned}
& R_{u}=\left\{r_{1}+. u, r_{2}+u, \ldots, r_{N}+u\right\}, \\
& D_{u}=v_{P}\left\{t_{1}, t_{2}, \ldots ., t_{N}\right\} .
\end{aligned}
$$

With the $\mathrm{N}$ by 4 matrix

$$
A_{u}=d R_{u} / d P_{u}
$$

the object is located by the iteration

$$
P_{u}^{p+1}=P_{u}^{p}+\left(A_{u}^{p T} W_{u} A_{u}^{p}\right)^{-1} A_{u}^{p T} W_{u}\left(R_{u}^{p}-D_{u}\right)
$$


If the errors in the $t_{n}$ are independent gaussian random variables with zero means, the maximum-likelihood, minimum-variance weighting matrix is simply

$$
\mathrm{w}_{\mathrm{u}}=\left[\begin{array}{lllll}
\mathrm{g}_{1} & & & \\
& & \mathrm{~g}_{2} & & \\
& 0 & & \ddots & \mathrm{g}_{\mathrm{N}}
\end{array}\right]
$$

where $g_{n}=1 / \nu_{n}$ and $\nu_{n}$ is the variance of the error in $v_{p} t_{n}$. Using this $w_{u}$, the covariance matrix of the location errors and the error in $u$ is $P_{u c}=B_{u}^{-1}$, where, as usual

$$
\begin{array}{r}
\mathrm{B}_{\mathrm{u}}-\Lambda_{\mathrm{u}}^{\mathrm{T}} \mathrm{W}_{\mathrm{u}} \mathrm{A}_{\mathrm{u}} \\
\quad \text { Comparison of TOA Errors and TDOA Errors }
\end{array}
$$

The second important result in this paper is now proved.

\section{Proposition 2}

If the maximum-likelihood, minimum-variance weighting matrix is used, the covariance matrix of the object location errors is the same whether TOA or TDOA methods are used.

Again, Proposition 2 is proved only if the TOA errors are independent random variables, with zero mean; but it is likely to be valid if the TOA errors are correlated.

We prove the proposition for the general case of M unknuwns and $N>$ M TOA iditititasurements for $\mathrm{N}$ sensors. Let there be $\mathrm{M}$ "position" unknowns $u_{m}, \mathrm{~m}=1,2, \ldots$, $M$, and let the "initial time" unknown be $u_{M+1}$; i.e., $u=u_{M+1}$. The $M+1$ dimensional vector of unknowns is

$$
P=\left\{u_{1}, u_{2}, \ldots, u_{M I}, u_{M+1}\right\}
$$

With TOA-type data from $N>M$ sensors, we wish to satisfy the $N$ equations

$$
r_{n}\left(u_{1}, \ldots, u_{M}\right)+u_{M+1}-d_{n}=0, n=, 2, \ldots, N
$$

in the least-squares sense, where $d_{n}$ is proportional to the data from the nth sensor and $r_{n}$ is a function that models $d_{n}$. Let

$$
A=\left(\alpha_{n m}\right) ; \alpha_{n m}=d\left(r_{n}+u_{M+1}\right) / d u_{m}, m=1, \ldots, M+1
$$


By analogy with the previous material we would use $u_{1}=x, u_{2}=y$, etc., $d_{n}=v_{p} t_{n}, u_{M+1}=v_{p} t_{0}$, and $\alpha_{n 1}=a_{n}, \alpha_{n 2}=b_{n}$, etc. As usual let the $d_{n}$ errors be independent random variables with zero mean and inverse variance $g_{n}$.

Now, define

$$
s=\sum_{n=1}^{N} g_{n}, \quad h_{m}=\sum_{n=1}^{N} g_{n} \alpha_{n m} \text {, and } f_{i j}=\sum_{n=1}^{N} g_{n} \alpha_{n i} \alpha_{n j} \text {. }
$$

For example, $h_{1}=h_{a}, f_{12}=f_{a b}$, etc.

The location error covariance matrix $P_{c}$ for the TDOA system is $M$ by $M$, and the $P_{u c}$ for the TOA method is $M+1$ by $M+1$. We now prove that the upper left $M-b y-M$ matrix of $\mathrm{B}_{\mathrm{u}}^{-1}=\mathrm{P}_{\mathrm{uc}}$ is identical to $\mathrm{B}^{-1}=\mathrm{P}_{\mathrm{c}}$.

Let the elements of $\mathrm{B}$ be $\beta_{\mathrm{ij}}$. Then, from Eq. (15),

$$
\beta_{i j}=f_{i j}-h_{i j} h_{j} / s \quad .
$$

If

$$
F=\left(f_{i j}\right) \text { and } H=\left\{h_{1}, h_{2}, \ldots, h_{M}\right\} \text {, }
$$

where $F$ is an $M-b y-M$ matrix, then $B$ is clearly of the form

$$
B=F-\mathrm{HH}^{\mathrm{T}} / \mathrm{s} \text {. }
$$

This form sonforms to that of Theorem 1 and thus

$$
\begin{aligned}
B^{-1} & =F^{-1}-\lambda F^{-1} H^{T} F^{-1} / s \\
& =F^{-1}+F^{-1} H^{T} F^{-1} /\left(s-H^{T} F^{-1} H\right) .
\end{aligned}
$$

Now consider $B_{u}$. We get

$$
B_{u}=\left[\begin{array}{ccc}
B_{u_{11}} & B_{u_{12}} \\
\hdashline B_{u_{12}}^{T} & B_{u_{22}}
\end{array}\right]=\left[\begin{array}{l:c}
F & H \\
\hdashline-1:- & s
\end{array}\right] \text {. }
$$


From Theorem 2,

$$
\mathrm{q}=\mathrm{s}-\mathrm{H}^{\mathrm{T}} \mathrm{F}^{-1} \mathrm{H}
$$

and

$$
\begin{aligned}
B_{u_{11}}^{-1} & =F^{-1}+\left(F^{-1} H\right)\left(H^{T} F^{-1}\right) / q \\
& =F^{-1}+F^{-1} H H^{T} F^{-1} /\left(s-H^{T} F^{-1} H\right) .
\end{aligned}
$$

Equation (20) is identical to Eq. (21) and the proof is complete.

\section{Conclusions}

It has been shown that the covariance matrix of object location errors is identical for TOA and all TDOA schemes if the maximum-likelihood, minimum-variance weighting matrix is used. This weighting is optimal if the TOA errors are jointly gaussian random variables. This description of the data errors is not generally restrictive. The TOA, TDOA identity has been proved only if the data errors are independent random variables, but we feel it is likely valid if the errors are correlated. In most applications these errors are not correlated.

The optimal weighting matrix is the inverse of the covariance matrix of the data crroro in the TOA case and the inverse of the covariance of the data error differences in the TDOA case. In any application the absolute values of the weighting matrix need not be known, but only their relative values are required for optimality.

At least in the situation where the data errors are taken as independent random variables it seems that a direct TOA scheme is simpler than any TDOA scheme. The TOA method requires the inversion of a matrix of order one higher than any TDOA method, but the elements of the TOA matrices are easier to compute than those of the TDOA. In the absence of evidence to the contrary we general'ly recommend the TOA method.

We have not examined considerations such as numerical analysis and programming problems in this report. In any implementation of TOA or TDOA, these kinds of considerations must be included in the decision as to system methods. 


\section{References}

1. E. A. Aronson, "Location Errors in Angle-Measuring and Distance-Measuring Systems," SAND77-0364, Sandia Laboratories, Albuquerque, NM, March 1977.

2. D. Graupe, Identification of Systems (New York: Van Nostrand-Reinhold Co., 1972), pp. 104-106.

3. S. M. Selby, Ed., CRC Standard Mathematical Tables, 23rd Ed., (Cleveland, Ohio: CRC Press, 1973), p. 124 .

4. R. T. Gregory and D. L. Karney, A Collection of Matrices for Testing Computational Algorithms (New York: Wiley-Interscience, 1969), p. 6. 


\section{DISTRIBUTION:}

AFTAC/TFS (2)

Patrick AFB, FL 32925

Attn: Maj. M. F. Schneider

SAMSO/SZSE (2)

P. O. Box 92960

Worldway Postal Center

Los Angeles, CA 90009

Attn: Maj. A. H. Hayden, Jr.

1240 H. M. Dumas

1241 D. E. Henry

1242 T. G. Taylor

1243 G. H. Mauth

1330 R. C. Maydew

1730 W. C. Myre

2600 L. E. Hollingsworth

2610 R. J. Detry

2613 M. R. Scott

2613 E. A. Aronson (10)

2630 E. K. Montoya

2640 J. L. Tischhauser

2644 A. R. Iacoletti

5122 R. J. Hanson

8150 D. E. Gregson

8410 R. Baroody

9400 H. E. Lenander

9410 R. L. Brin

9411 E. White

9420 T. L. Pace

9422 G. L. West

9424 F. D. Gutierrez

9426 J. L. Rogers

y470 S. A. Moore

9471 R. D. Bentley

9473 J. D. Patrick

948ก T. S. Ghuroh

Y483 W. V. Hereford

9520 R. H. Schultz

8266 E. A. Aas

3141 C. A. Pepmueller (Actg) (5)

3151 W. L. Garner (3)

For: ERDA/TIC (Unlimited Release)

ERDA / TIC (25)

(R. P. Campbell, 3172-3) 